\title{
L-Cordial Labeling for Path Union Graphs of Flywheel of 4 Stroke Single Cylinder Engine
}

\section{J. Arthy}

Abstract: In this work we prove that path union of $\mathrm{H}_{n}$, duplication of all edge of $H_{n}, \quad$ Cube $Q_{3}$, Octahedron, $P\left(H_{n} \odot \overline{K_{1, m}}\right)$, Hanging $H_{n}$,Hanging Cube $Q_{3}$, $P((r, H($ octohedron $)), \quad$ Graphs admits L-Cordial Labeling.

Keywords: $H_{n}$ graphs, Path Union, Hanging graph, Cube $Q_{3}$, Octahedron graphs, L-Cordial Labeling (LCL).

AMS: Classification: 05C78.

\section{INTRODUCTION}

In this current assessment we make use of graph $G=(p, q)$ which are finite, simple and undirected. Primarily $L$-Cordial Labeling (LCL) being introduced in [8] and immaculately certain Graphs provided to have a diverse range of standards that proved for the same $[9,10]$ LCL. In $[2,3]$ et al proved cube $Q_{3}$, octahedron and special graphs also confesses LCL. $H$ - Related graph for square difference, prime cordial and Cube difference labeling were studied in $[1,7,13]$. Hanging and path union of pyramid graph for SSL and SDL was proved in [6,12].S.K.Vaidya and U.M. Prajapati, [11,14] have proven to be prime labeling of duplication of $K_{1, n}, C_{n}$ graph and super mean labeling of $\mathrm{Q}_{3}$, Octahedron, and some families of graphs were proved. Detailed survey descriptions are given in [4]. Condition and results follow from [5] in this work we prove path union of some special graphs admits $L$-Cordial labeling.

\section{PRELIMINARIES}

1. Graph $G(V, E)$ has $L$-cordial labeling if there is a bijection function $f: E(G) \rightarrow\{1,2, \ldots|E|\}$ Thus the vertex label is induced as 0 if the biggest label on the incident edges is even and is induced as 1 , if it is odd. The condition is satisfied further by $V_{f}(0)$ which number of vertices labeled with 0 and $V_{f}(1)$ which is the number of vertices labeled with 1 , and follows the condition that $\left|V_{f}(1)-V_{f}(1)\right| \leq 1$. isolated vertices are not included for labeling here. An $L$-cordial graph is a graph which admits the above labeling.

Revised Manuscript Received on December 11, 2019

J. Arthy, Assistant Professor, Department of Science and Humanities, Bharath Institute of Higher Education and Research, India
2. Hanging graph is obtained by attaching a graph $G$ to a new pendent edge and is denoted by $H(G)$.

3. An octahedron is a polyhedron with 8 faces.

4. The graph $P_{2} \times P_{2} \times P_{2}$ is called the cube and is denoted by $Q_{3}$.

5. $H_{n}$-graph obtained from two copies of path with vertices $a_{1}, a_{2}, \ldots, a_{n}$ and $b_{1}, b_{2}, \ldots, b_{n}$ by connecting the vertices $a_{\frac{n+1}{2}}$ and $b_{\frac{n+1}{2}}$ if $n$ is odd and $b_{\frac{n}{2}+1}$ and $a_{\frac{n}{2}}$ is joined if $n$ is even.

Theorem 2.1

For $(n \geq 3) P\left(r, H_{\mathrm{n}}\right)$ is L-Cordial Graph.

Proof:

Consider $G$ with $V=\left\{x_{i}{ }^{(j)}, y_{i}{ }^{(j)} ; 1 \leq i \leq n, 1 \leq j \leq r\right\}$ and the edge set

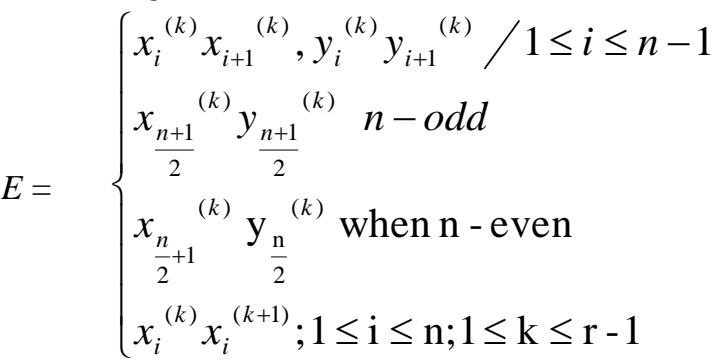

Define a $f: E(G) \rightarrow\{1,2 \ldots|E|\}$ as follows;

For $1 \leq i \leq n-1,1 \leq k \leq r$

$f\left(x_{\mathrm{i}}{ }^{(k)} x_{i+1}{ }^{(k)}\right)=2 r+2 i-2+2(n-1)(k-1)$

$f\left(y_{i}{ }^{(k)} y_{i+1}{ }^{(k)}\right)=2 r+2(i-1)+2(n-1)(k-1)+1$

$f\left(x_{1}{ }^{(k)} x_{1}{ }^{(k+1)}\right)=k, 1 \leq \mathrm{k} \leq \mathrm{r}-1$

For $1 \leq \mathrm{k} \leq \mathrm{r}$

$f\left(x_{\frac{\mathrm{n}+1}{2}}{ }^{(k)} y_{\frac{n+1}{2}}{ }^{(k)}\right)=r+(k-1), \quad \mathrm{n}$ is odd

$f\left(x_{\frac{\mathrm{n}}{2}+1}{ }^{(k)} y_{\frac{n}{2}}{ }^{(k)}\right)=r+k-1, \mathrm{n}$ is even

Thus the above edge labeling satisfies the condition of $L$-Cordial labeling and $V_{f}(0)=V_{f}(1)$ for $n \geq 3$.

Hence $P\left(r, H_{n}\right)$ is L-Cordial Graph. 


\section{L-Cordial Labeling for Path Union Graphs of Flywheel of 4 Stroke Single Cylinder Engine}

\section{Theorem 2.2}

The Path union $r$ copies of duplication of all edge of $H_{n}$ graph for $(n \geq 3)$ admit $L$ - Cordial labeling.

\section{Proof:}

Let $G=(V, E)$ be an $H_{n}$ graph obtained by duplicating of the all edges ,then we define a edge labeled function $g: E \rightarrow\{1,2, \ldots .(6 n r-2 r-1)\}$ as follows;

$$
\begin{aligned}
& \text { for } 1 \leq i \leq n-1,1 \leq t \leq r \\
& g\left(u_{i}{ }^{(t)} u_{i+1}{ }^{(t)}\right)=r+2 i+1+(6 n-3)(t-1) \\
& g\left(v_{i}{ }^{(t)} v_{i+1}{ }^{(t)}\right)=r+2 i+5-3 t+6(n t-n) \\
& g\left(x_{i}{ }^{(t)} u_{i+1}{ }^{(t)}\right)=r+2 n+4 i+(6 n-3)(t-1)-1 \\
& g\left(y_{i}{ }^{(t)} v_{i+1}{ }^{(t)}\right)=r+2 n+4 i+(6 n-3)(t-1) \\
& F o r 1 \leq \mathrm{i} \leq \mathrm{n}, 1 \leq \mathrm{t} \leq \mathrm{r} \\
& \mathrm{g}\left(\mathrm{x}_{\mathrm{i}}{ }^{(t)} u_{i}{ }^{(t)}\right)=r+2 n+4 i-3+(6 n-3)(t-1) \\
& g\left(y_{i}{ }^{(t)} v_{i}{ }^{(t)}\right)=r+2 n+4 i-2+(6 n-3)(t-1)
\end{aligned}
$$

When $n$-even

$$
\begin{aligned}
& g\left(u_{\frac{n}{2}+1}^{(t)} w^{(t)}\right)=r+4-3 t+6 n(t-1) \\
& g\left(u_{\frac{n}{2}+1}{ }^{(t)} v_{\frac{n}{2}}{ }^{(t)}\right)=r+(6 n-3)(t-1) \\
& g\left(v_{\frac{n}{2}}{ }^{(t)} w^{(t)}\right)=r-3 t+5+6 n t-6 n \\
& \text { When } n \text { - odd } \\
& g\left(u_{\frac{n+1}{2}}^{(t)} w^{(t)}\right)=r+4-3 t+6 n(t-1) \\
& g\left(u_{\frac{n+1}{2}}^{(t)} v_{\frac{n+1}{2}}^{(t)}\right)=r+(6 n-3)(t-1) \\
& g\left(v_{\frac{n+1}{2}}^{(t)} w^{(t)}\right)=r-3 t+5+6 n(t-1)
\end{aligned}
$$

From the above labeling it is clear that path union of Duplication all edge of $H_{n}$ - graph satisfies the condition $v_{g}(0)=v_{g}(1)$

Hence the graph admits $L$ - Cordial labeling.

\section{Theorem 2.3}

Path union r copies of Cube $Q_{3}$ graph is $L$-Cordial Graph.

\section{Proof:}

$$
\text { Let } G=P\left(r, \text { Cube } Q_{3}\right) \text { Graph, with vertices }
$$

$V=\left\{u_{i}{ }^{(k)}, v_{i}{ }^{(k)} / 1 \leq i \leq 4,1 \leq k \leq r\right\}$ and the edge set $E=\left\{u_{i}{ }^{(k)} u_{i+1}{ }^{(k)}, v_{i}{ }^{(k)} v_{i+1}{ }^{(k)} / 1 \leq i \leq 3,1 \leq k \leq r\right\}$ $\cup\left\{u_{i}{ }^{(k)} v_{i}{ }^{(k)}, 1 \leq i \leq 4,1 \leq k \leq r\right\} \cup\left\{v_{1}{ }^{(k)} v_{4}{ }^{(k)}, u_{1}{ }^{(k)} u_{4}{ }^{(k)}\right\}$

Then $|V(G)|=8+r$-1and $|E(G)|=13 \mathrm{r}-1$

Define an edge labeled function

$f: E(G) \rightarrow\{1,2, \ldots,(13 r-1)\}$ as

$f\left(u_{1}{ }^{(k)} u_{1}{ }^{(K+1)}\right)=k ;$ For $1 \leq \mathrm{k} \leq \mathrm{r}-1$

For $1 \leq i \leq 3,1 \leq k \leq r-1$

$f\left(\mathrm{u}_{\mathrm{i}}{ }^{(k)} u_{i+1}{ }^{(k)}\right)=r+i+12 k-13$

$f\left(v_{i}{ }^{(k)} v_{i+1}{ }^{(k)}\right)=r+i+12 k-9$

$f\left(u_{i}{ }^{(k)} v_{i}{ }^{(k)}\right)=r+i+12 k-5 ; 1 \leq \mathrm{i} \leq 4,1 \leq \mathrm{k} \leq \mathrm{r}$

$f\left(\mathrm{u}_{1}{ }^{(k)} u_{4}{ }^{(k)}\right)=r+12 k-9$

$f\left(v_{1}{ }^{(k)} v_{4}{ }^{(k)}\right)=r+12 k-5$

Hence, we have $V_{f}(0)=V_{f}(1)=4 r$.

Therefore $P\left(r\right.$, Cube $\left.Q_{3}\right)$ is L- Cordial Graph.

Theorem 2.4

The graph $P(r$,Octahedron $)$ admits $L$-Cordial Labeling.

\section{Proof:}

Consider graph $G$, be the path union of $r$ Copies of Octahedron.

Let $v=\left\{x_{j}{ }^{(t)}, y_{j}{ }^{(t)} ; 1 \leq j \leq 3\right\}$ be a Vertex set and

$E=\left\{x_{j}{ }^{(t)} y_{j}{ }^{(t)} / 1 \leq j \leq 3,1 \leq t \leq r\right\} \cup$

$\left\{x_{j}{ }^{(t)} x_{j+1}{ }^{(t)}, y_{j}{ }^{(t)} y_{j+1}{ }^{(t)}, x_{j}{ }^{(t)} y_{j+1}{ }^{(t)}, x_{1}{ }^{(t)} x_{3}{ }^{(t)}, y_{1}{ }^{(t)} y_{3}{ }^{(t)} / 1 \leq j \leq 2,1 \leq t \leq r\right\}$

$\cup\left\{x_{1}^{(t)} x_{1}^{(t+1)} \quad 1 \leq \mathrm{t} \leq \mathrm{r}-1\right\}$.

Then the Labeling of bijective function,

$g: E(G)--\{1,2, \ldots|E|\}$ is defines as follow

$g\left(x_{1}^{(t)} x_{1}^{(t+1)}\right)=t ; 1 \leq \mathrm{t} \leq \mathrm{r}-1$

for $1 \leq \mathrm{t} \leq \mathrm{r}$

$$
\begin{aligned}
& g\left(x_{j}^{(t)} y_{j}^{(t)}\right)=r+2 j+12 t-13 \\
& g\left(x_{1}^{(t)} x_{3}^{(t)}\right)=r+12 t-1 \\
& g\left(y_{1}^{(t)} y_{3}^{(t)}\right)=r+12 t-4 \\
& g\left(x_{j}^{(t)} y_{j}^{(t)}\right)=r+2 j+12 t-3, \quad 1 \leq \mathrm{j} \leq 2
\end{aligned}
$$

For $1 \leq \mathrm{j} \leq 2,1 \leq \mathrm{t} \leq \mathrm{r}$

$$
\begin{aligned}
& \mathrm{g}\left(\mathrm{x}_{\mathrm{j}}^{(\mathrm{t})} x_{j+1}^{(t)}\right)=r+12 t+j-4 \\
& g\left(y_{j}^{(t)} y_{j+1}^{(t)}\right)=r+12 t+j-7 \\
& g\left(x_{j+1}^{(t)} y_{j}^{(t)}\right)=r+12 t+2 j-12
\end{aligned}
$$

In the view of above labeling it is made clear that $V_{g}(0)=$ $V_{g}(1)=3$ r. Thus path union of the graph admits $L$ - Cordial labeling. Hence $L$-Cordial Graph.

\section{Theorem 2.5}

Path union of hanging $\mathrm{H}_{\mathrm{n}}$ graph is $L$-Cordial Graph. 


\section{Proof:}

Consider graph $G$ to be path union of hanging $H_{n}$ graph with vertices,

$$
\begin{aligned}
& v=\left\{u_{i}^{k}, v_{i}^{k}, w^{k} ; 1 \leq k \leq r, 1 \leq i \leq n\right\} \text { and edges } \\
& E=\left\{u_{i}^{k} u_{i+1}^{k}, v_{i}^{k} v_{i+1}^{k}, w^{k} u_{1}^{k} ; 1 \leq i \leq n-1,1 \leq k \leq r\right\} \\
& \cup\left\{w^{k} w^{k+1} / 1 \leq k \leq r-1\right\} \\
& \cup\left\{u_{\frac{n+1}{2}}^{(k)} v_{\frac{n+1}{2}}^{(k)} \text { when } n\right. \text { is odd and } \\
& \left.u_{\frac{n}{2}+1}^{k} v_{\frac{n}{2}}^{k} \text { when } n \text { is even }\right\}
\end{aligned}
$$

We define $f: E \rightarrow q$ as follows

For $i=1, \ldots, n-1, k=1,2, \ldots, r$

$f\left(u_{i}^{k} u_{i+1}^{k}\right)=2 r+1+2 i-2+(2 n-1)(k-1)$

$f\left(v_{i}^{k} v_{i+1}^{k}\right)=2 r+2+2(i-1)+(2 n-1)(k-1)$

$f\left(w^{k} w^{k+1}\right)=k ; 1 \leq i \leq r-1$

for $k=1,2, \ldots, r$

$f\left(w^{k} u_{1}^{k}\right)=k+1$

$f\left(u_{\frac{n+1}{2}}^{k} v_{\frac{n+1}{2}}^{k}\right)=2 r+(2 n-1)(k-1)$ for $n$ odd

$f\left(u_{\frac{n}{2}+1}^{k} v_{\frac{n}{2}}^{k}\right)=2 r+(2 n-1)(k-1)$ for $n$ even

Thus $v_{f}(0)+1=v_{f}(1) r$ is odd and $v_{f}(0)=v_{f}(1)$

When $r$ Even for all $(n \geq 3)$.

Therefore path union of hanging $H_{n}$ graph is $L C G$.

\section{Theorem 2.6}

The Graph $P\left(H_{n} \odot \overline{K_{1 m}}\right)$ admits L- Cordial Labeling For $n \geq 3, m \geq 2$

\section{Proof:}

Let $G=P\left(H_{n} \odot \overline{K_{1 m}}\right)$

with

$V(G)=\left\{u_{i}^{k}, v_{i}^{k}, u_{i j}^{k}, v_{i j}^{k} / i=1,2, \ldots ., n, j=1, . . m, k=1,2\right.$,
And

$$
E(G)=\left\{\begin{array}{l}
u_{i}^{k} u_{i+1}^{k}, v_{i}^{k} v_{i+1}^{k} / 1 \leq i \leq n-1,1 \leq k \leq r \\
u_{i}^{k} u_{i j}^{k}, v_{i}^{k} v_{i j}^{k} / 1 \leq i \leq n, 1 \leq j \leq m, 1 \leq k \leq r \\
u_{1}^{k} u_{1}^{k+1} / 1 \leq k \leq r-1 \\
\text { For } 1 \leq k \leq r \\
u_{\frac{n+1}{2}}^{k} v_{\frac{n+1}{2}}^{k} \text { when } n \text { is odd } \\
u_{\frac{n}{2}+1}^{k} v_{\frac{n}{2}}^{k} \text { when } n \text { is even }
\end{array}\right.
$$

We define a map $f: E \rightarrow\{1,2, \ldots|E|\}$ as follows

For $1 \leq i \leq n, 1 \leq j \leq m, 1 \leq k \leq r$

$$
\begin{aligned}
g\left(u_{i}^{k} u_{i j}^{k}\right)= & 2 r+2(j-1)+2 m n(k-1)+4(i-1) \\
& +2(m-2)(i-1) \\
g\left(v_{i}^{k} v_{i j}^{k}\right)= & 2 r+2 j+2 m(n k+n+i-1)-1
\end{aligned}
$$

For $i=1,2, \ldots n-1, k=1,2 \ldots . r$

$$
g\left(u_{i}^{k} u_{i+1}^{k}\right)=2 r(m n+1)+(2 n-2)(k-1)+2(i-1)
$$$$
g\left(v_{i}^{k} v_{i+1}^{k}\right)=2 r(m n+1)+(2 n-2)(k-1)+2 i-1
$$

For $1 \leq k \leq r-1$

$g\left(u_{1}^{k} u_{1}^{k+1}\right)=k$

For $1 \leq k \leq r$

$g\left(u_{\frac{n+1}{2}}^{k} v_{\frac{n+1}{2}}^{k}\right)=r+k-1 \quad$ when $n$ is Odd $g\left(u_{\frac{n}{2}+1}^{k} v_{\frac{n}{2}}^{k}\right)=r+k-1 \quad$ when $n$ is Even

From the above labeling it is clear that $V_{g}(0)=V_{g}(1)$ for all $n \geq 3, m \geq 2$. Thus $P\left(H_{n} \odot \overline{K_{1 m}}\right)$

admits $L$-Cordial labeling.

\section{Theorem 2.7}

Path Union of Hanging Cube $Q_{3}$ graph admits L-Cordial Labeling.

\section{Proof:}

Consider $G=(V, E)$ be a path union of $r$ copies of Hanging Cube $Q_{3}$ with

$$
\begin{aligned}
& V=\left\{u_{j}^{t}, v_{j}^{t}, w^{t} / 1 \leq j \leq 4,1 \leq t \leq r\right\} \text { and } \\
& E=\left\{u_{j}^{t} u_{j+1}^{t}, v_{j}^{t} v_{j+1}^{t} / 1 \leq j \leq 3,1 \leq t \leq r\right\} \\
& \cup\left\{u_{j}^{t} v_{j}^{t}, v_{1}^{t} v_{4}^{t}, u_{1}^{t} u_{4}^{t}, w^{t} u_{1}^{t} / 1 \leq j \leq 4,1 \leq t \leq r\right\} \\
& \cup\left\{w^{t} w_{1}^{t+1}, 1 \leq t \leq r-1\right\}
\end{aligned}
$$

Define

$f: E(G) \rightarrow\{1,2 \ldots q\}$ as follows 


\section{L-Cordial Labeling for Path Union Graphs of Flywheel of 4 Stroke Single Cylinder Engine}

For $j=1,2,3$ and $t=1,2 \ldots r$

$f\left(u_{j}^{t} u_{j+1}^{t}\right)=2(r+6 t)+j-13$

$f\left(v_{j}^{t} v_{j+1}^{t}\right)=2 r+12 t+j-9$

$f\left(w^{t} u_{1}^{t}\right)=k+t-1$

$f\left(v_{4}^{t} v_{1}^{t}\right)=2 r+12 t-5$

$f\left(u_{4}^{t} u_{1}^{t}\right)=2 r+12 k-9$

For $1 \leq j \leq 4,1 \leq t \leq r$

$f\left(u_{j}^{t} v_{j}^{t}\right)=2 r+12 k-5+i$

For $1 \leq t \leq r-1$

$f\left(w^{t} w^{t+1}\right)=t$

Hence from the above labeling, it is clearly seen that $V_{f}(0)=V_{f}(1) \quad$ when $\quad \mathrm{r} \quad$ is even and $V_{f}(1)=V_{f}(0)+1$ when $\mathrm{r}$ is odd. Thus Path union of r-copies of Hanging Cube $Q_{3}$ graph admits LCL.

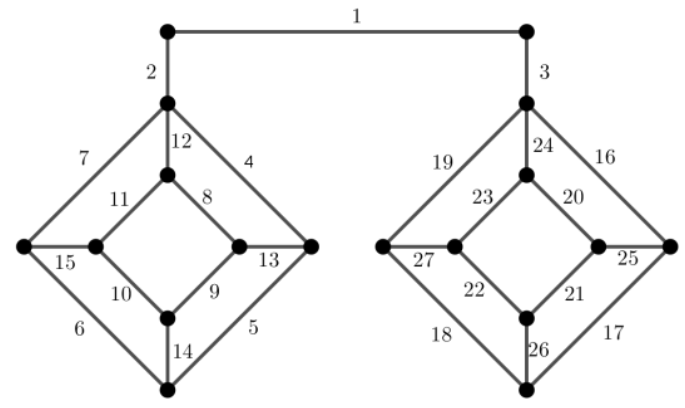

2 Copies of Hanging Cube $Q_{3}$

\section{Theorem 2.8}

Path union of r-copies of Hanging Octahedron graph admits LCL.

\section{Proof:}

Let $\mathrm{G}$ be $P((r, H$ (octohedron $))$ graph with

$$
\begin{aligned}
& V(G)=\left\{a_{i}^{k}, b_{i}^{k}, x^{k} / 1 \leq i \leq 3,1 \leq k \leq r\right\} \text { and } \\
& E(G)=\left\{a_{i}^{k} a_{i+1}^{k}, b_{i}^{k} b_{i+1}^{k}, b_{i}^{k} a_{i+1}^{k}, a_{1}^{k} a_{3}^{k}, b_{1}^{k} b_{3}^{k}\right. \\
& \quad / 1 \leq i \leq 21 \leq k \leq r\} \\
& \cup\left\{x^{k} x^{k+1}, 1 \leq k \leq r-1\right\} \cup\left\{a_{i}^{k} x^{k}, 1 \leq i, k \leq r\right\}
\end{aligned}
$$

Then the Labeling of Bijective function

$f: E(G) \rightarrow\{1,2, \ldots|E|\}$ is defined as

$$
\begin{aligned}
& f\left(a_{i}^{k} a_{i+1}^{k}\right)=2 r+12 k+i-1, \text { For } i=1,2 k=1,2, \ldots r \\
& f\left(b_{i}^{k} b_{i+1}^{k}\right)=2 r+12 k+i-7 \\
& f\left(b_{i}^{k} a_{i+1}^{k}\right)=2 r+12 k+i-11 \\
& f\left(b_{3}^{k} a_{1}^{k}\right)=2 r+12 k-12
\end{aligned}
$$

$$
\begin{aligned}
& f\left(a_{1}^{k} a_{3}^{k}\right)=2 r+12 k-1 \\
& f\left(b_{3}^{k} b_{1}^{k}\right)=2 r+12 k-4 \\
& f\left(a_{1}^{k} x^{k}\right)=r+k-1 \\
& \text { For } 1 \leq k \leq r-1 \\
& f\left(x^{k} x^{k+1}\right)=k
\end{aligned}
$$

From the above labeling. It is clear that $V_{f}(0)=V_{f}(1)$, when $\mathrm{n} \equiv 0(\bmod 2)$ and $V_{f}(1)=V_{f}(0)+1$,

When $\mathrm{n} \equiv 1(\bmod 2)$ and satisfies the condition of LCL. Thus $P(r, H$ (octohedron $)$ is LCG.

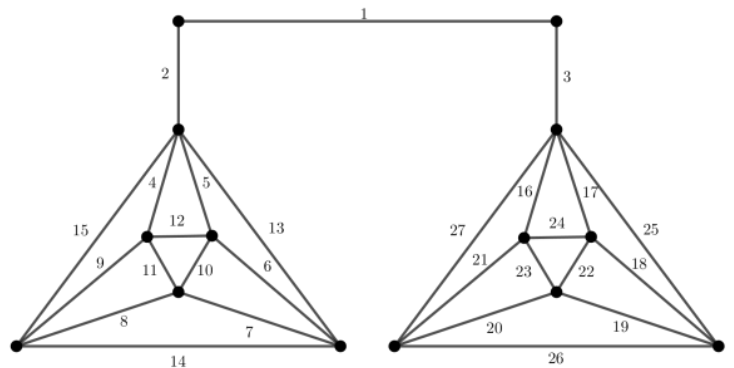

2 Copies of Hanging Octahedron

\section{CONCLUSION}

In this paper we have established that path union of $H_{\mathrm{n}}$, duplication of all edge of $H_{n}$, Cube $Q_{3}$, Octahedron, $P\left(H_{n} \odot \overline{K_{1 m}}\right)$, Hanging $H_{\mathrm{n}}$, Hanging Cube $Q_{3}$, $P((r, H$ (octohedron $))$ are $L$ - Cordial graphs.

\section{REFERENCES}

[1] J.Arthy, K.Manimekalai and K.Ramanathan, "Cube Difference Labeling Of Some Special Graphs”. AIP Conference Proceedings 2112, 020140 (2019).

[2] J.Arthy, K.Manimekalai and K.Ramanathan, "Cycle And Path Related Graphs on L-Cordial Labeling". International journal of Innovative Science and Research Technology, Vol 4, issue 5, May 2019, ISSN No: 2456-2165.

[3] J.Arthy, K.Manimekalai, K.Bhuvaneswari "On L-Cordial Graphs", Advancement in Engineering, Science and Technology. J.Mech.Cont.AMath.Sci, Special issue, N0-2, August (2019) PP 647-652.

[4] Gallian. J.A.“A dynamic survey of graph labeling”, The Electronics journal of Combinatories, 17(2010) \#DS6.

[5] F. Harary. "Graph theory" Narosa Publishing House (2001).

[6] P.Jagadeeswari, K.Ramanathan and K.Manimekalai "Square Difference Labeling for Pyramid Graph and its Related Graphs", Introduction Journal Of Mathematics and its application, Int.J.Math And Appl,6(1)2018,91-96.

[7] P.Jagadeeswari, K.Manimekalai and K.Ramanathan,, "Square Difference Labeling Of $H$-Graphs" AIP Conference Proceedings 2112, 020147 (2019).

[8] Mukund V.Bapat, "Some Vertex Prime Graphs and A New Type Of Graph Labeling", International Journal Of Mathematics Trends and Technology (IJMTT), Vol 47, Number 1(2017), 49-55. 
[9] Mukund, V.Bapat, "A Note On L-Cordial Labeling Of Graphs", International Journal Of Mathematics And Its Applications, Vol 5, Issue 4-D(2017),457-460.

[10] Mukund V.Bapat, "New Results On L-Cordial Labeling Of Graphs", International Journal Of Statistics And Applied Mathematics, 2018, 3(2), 641-644.

[11] Dr.A.Selvam Avadayappan, R.Sinthu,"New families of super mean graphs", International Journal of Scientific and Engineering Research, Vol.7, Issue 3(2013), 692-697.

[12] G.Subhashini, K.Ramanathan and K.Manimekalai, "Square Sum Labeling for Pyramid Graph and Hanging Pyramid Graph", Introduction Journal Of Mathematics and its application, Int.J.Math And Appl, 6(1)(2018),97-102

[13] G.Subhashini, K.Manimekalai and K.Ramanathan, "Prime Cordial Labeling Of $H_{n} \odot K_{2}$ Graphs", AIP Conference Proceedings 2112, 020128 (2019).

[14] S.K. Vaidya and U.M. Prajapati, "Prime Labeling in the context of duplication of graph elements", International Journal of Mathematics and soft computing, Vol.3.No.1 (2013)13-20.

\section{AUTHORS PROFILE}

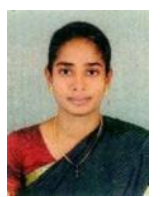

P. Jagadeeswari is an Assistant Professor, Department of Science and Humanities, BIHER, Chennai. She is currently working on Graph labeling. She published 7 papers in international journal. 